\title{
Adaptive Matching of the Radar Signal and Image Display Device Dynamic Ranges
}

\author{
Vitaliy Garmash \\ Department of Radio Electronic Systems, \\ Baltic State Technical University «VOENMEH», \\ St. Petersburg, 190005, Russia \\ Corresponding author: gar-vitalij@yandex.ru \\ Yuriy Petrov \\ Department of Radio Electronic Systems, \\ Baltic State Technical University «VOENMEH», \\ St. Petersburg, 190005, Russia \\ E-mail: petrov-i4@yandex.ru
}

\author{
Andrey Andreev \\ Department of Technosphere Safety, \\ Peter the Great St. Petersburg Polytechnic University, \\ St. Petersburg, 195220, Russia \\ Anatoly Zaitsev \\ Department of Technosphere Safety, \\ Peter the Great St. Petersburg Polytechnic University, \\ St. Petersburg, 195220, Russia \\ E-mail: zajtsev_ai@spbstu.ru
}

(Received December 5, 2018; Accepted September 13, 2019)

\begin{abstract}
This article presents nonlinear radar signal processing method to form an image of the Earth's surface. The method proposes to match the dynamic ranges of the received signal and of the visualization device. The essence of the method is adaptive nonlinear signal processing, which provides better local contrast of radar images and improves discrimination of individual objects. The computational complexity of the proposed algorithm is optimized and allows real-time implementation in the airborne computing systems with limited computational power. Objects with large RCS merged into large illuminated "spots"; their visibility on the surrounding background has been reduced, unwanted effects are due to the fact that the above algorithms have a single point effect. To overcome the problems, the «Retinex» algorithm is usually used. They do not take into account the local neighborhood of pixels; therefore, in cases where the image contains both highly dark and strongly light local areas, these algorithms cannot provide high-quality matching of dynamic ranges.
\end{abstract}

Keywords- Radar signal, Radar cross-section, Digital image processing, Nonlinear processing, Adaptive contrasting.

\section{Introduction}

The most convenient for human perception source of information about the real objects are their optical images. Such images are obtained by perspective projection of reflected electromagnetic waves in the visible spectrum. This is how the human eye sees. That is why optical sensors with different technical characteristics are the basis of helicopter optoelectronic systems (OES). OES are designed to support piloting and landing of a helicopter, observing of the surrounding space and detecting the objects of interest (Logunov et al., 2018). However, the quality of obtained optical 
International Journal of Mathematical, Engineering and Management Sciences

Vol. 4, No. 6, 1448-1458, 2019

https://dx.doi.org/10.33889/IJMEMS.2019.4.6-114

images depends on the conditions of illumination and transparency of the atmosphere, which significantly complicates their use at night, as well as in fog, rain, snow. These factors are the most critical for the OES as part of the search and rescue operations complexes (SROC) (Matveev et al., 2018; Bataev, 2019), which has to operate in all-weather, all-season conditions, in difficult climatic zones, such as Arctic. To overcome these shortcomings in the OES, other spectral ranges are used in conjunction with the optical sensors (cameras). For example, fusion of images generated by television and infrared imaging sensors has found wide application in advanced vision systems used in aviation (EUROCAE ED, 2011; Shelton et al., 2012; Vygolov, 2013; Zheltov and Vygolov, 2015). The thermal sensor reacts on the temperature difference between the observed object and the surrounding background. This can be not enough for SROC, as there may be no such a temperature difference, e.g. in the case of searching for a life raft in a coastal marine area. The solution of such a problem becomes possible if the radar is included in the SROC (Ivanov et al., 2015; Davydov et al., 2016; Kaasik and Rogov, 2016; Ivanov et al., 2018).

Radars allow obtaining images of the earth's surface and objects located on it, regardless of the time of day and the level of illumination, in any weather conditions and at long ranges of observation. Radar sensors allow solving the problem of detection and recognition of stationary and moving small objects, including hidden by vegetation and snow cover. Millimeter-range radars are used to detect small objects on the sea surface and in coastal marine areas (Korobeynikov et al., 2016; Ziniakov et al., 2016; Shang et al., 2017; Klochkov et al., 2018). However, radar sensors also have a number of disadvantages compared to optical ones. These disadvantages include lower resolution and significant variation of the resulting objects image with the aspect angle.

\section{Radar Signal Reception}

Unlike television and thermal imaging, radar sensors are active. They independently generate and transmit electromagnetic waves towards the objects present in the observed scene and register the reflected wave to form a radar image of the targets. In this case, the received power of the radio signal is given by the radar equation (Skolnic, 1990; Tarasenko et al., 2017; Galeeva et al., 2018):

$P_{r}=\frac{P_{t} \cdot G_{t} \cdot A_{r} \cdot \sigma \cdot F^{4}}{(4 \cdot \pi)^{2} \cdot R^{4}}$

where $P_{t}$ is transmit power, $G_{t}$ - transmitting antenna gain, $A_{r}$ - aperture of the receiving antenna, $\sigma$ - radar cross-section (RCS) of the observed object, $F$ - signal propagation loss factor, $R-$ the distance between the radar and the observed object. The values $P_{t}, G_{t}, A_{r}$ and $F$ are constant for each radar image of the earth's surface since they are determined by the radar parameters and the propagation medium of the wave. The impact of the distance $R$ within the field of view is usually accounted for with the temporary automatic gain control (Meena and Prakasam, 2008). Thus, the only parameter that determines the appearance of the objects in observed scene when forming a two-dimensional radar image is the RCS of the objects.

RCS is a quantitative measure of the object property to dissipate electromagnetic waves. Large RCS values mean great radar visibility of the object; objects with low RCS are difficult to detect (Privalov and Shemanin, 2017, 2018). RCS of a particular object depends on its shape, size, material from which it is made, its orientation in relation to the radar antenna, and the radar wavelength. 
International Journal of Mathematical, Engineering and Management Sciences

Vol. 4, No. 6, 1448-1458, 2019

https://dx.doi.org/10.33889/IJMEMS.2019.4.6-114

\section{The Problem of Matching the Dynamic Ranges of the Radar Signal and Visualization Device}

RCS of the objects, which are of interest for surveillance radar, can vary by several orders of magnitude. For example, the RCS of a boat is $50 \mathrm{~m} 2$ and the RCS of carrier aircraft is $5000 \mathrm{~m} 2$; the RCS of a car is $5 \mathrm{~m} 2$ and the RCS of a bridge is $1000 \mathrm{~m} 2$. In the same way, the power of the reflected signal will differ, when it is received by the radar antenna. In practice, a situations when a few such objects are present simultaneously in the field of view often occurs. It is highly desirable to form a radar image in such a way that all objects are all clearly visible to the human eye, that is, have a sufficient level of local contrast. Modern graphics controllers, which are the part of avionics systems, use up to 24 bits to store and display information on the color of one pixel of the video image on the monitor. Radar images are monochrome and are usually rendered in shades of gray. The entire range of brightness values of each pixel is limited to 8 bits. Hence, the problem arises: how to match the power range of the received signal, which differs in the field of view by thousands of times, with 256 gradations used to display a monochrome image on the monitor? Linear matching from minimum to maximum (auto level) yields very poor results, so the standard way to solve the problem is a statistical approach (Figure 1). This method calculates the average power reflected from each of the $N$ resolution elements with linear coordinates $(x, y)$ of the signal $P_{r}(x, y)$ :

$\overline{\mathrm{P}_{\mathrm{r}}}=\frac{1}{\mathrm{~N}} \cdot \sum_{\mathrm{x}, \mathrm{y}} \mathrm{P}_{\mathrm{r}}(\mathrm{x}, \mathrm{y})$,

its standard deviation:

$\mathrm{SD}_{\mathrm{r}}=\sqrt{\frac{\sum_{\mathrm{x}, \mathrm{y}}\left(\mathrm{P}_{\mathrm{r}}(\mathrm{x}, \mathrm{y})-\overline{\mathrm{P}_{\mathrm{r}}}\right)^{2}}{\mathrm{~N}}}$

and produces a linear agreement on the power range

$\left(\overline{\mathrm{P}_{\mathrm{r}}}-3 \mathrm{SD}_{\mathrm{r}} ; \overline{\mathrm{P}_{\mathrm{r}}}+3 \mathrm{SD}_{\mathrm{r}}\right)$

of the received antenna signal with a range of visualization device. Those elements of the resolution, the signal from which is beyond the lower or upper limits of range (4), are assigned, respectively, the minimum or maximum brightness in the image.

A statistical approach for matching the dynamic range makes it possible to obtain convenient for the human eye radar images of the Earth's surface. However, if there are large conglomerates of objects with large RCS in the frame, some objects may be difficult to distinguish. This is the case even if these objects are located far beyond such conglomerates and have sufficient radio contrast to detect them in comparison with the background. The reason for this phenomenon is that in the process of statistical analysis, the power of the reflected signal from such objects is close to the lower limit of the range (4), or even beyond it. Thus, in the images from Figure 1 on the background of the water surface with a low RCS, there are a number of radio-contrast objects: a bridge, ships, and islands. However, due to the above reasons to distinguish them visually is very difficult. 

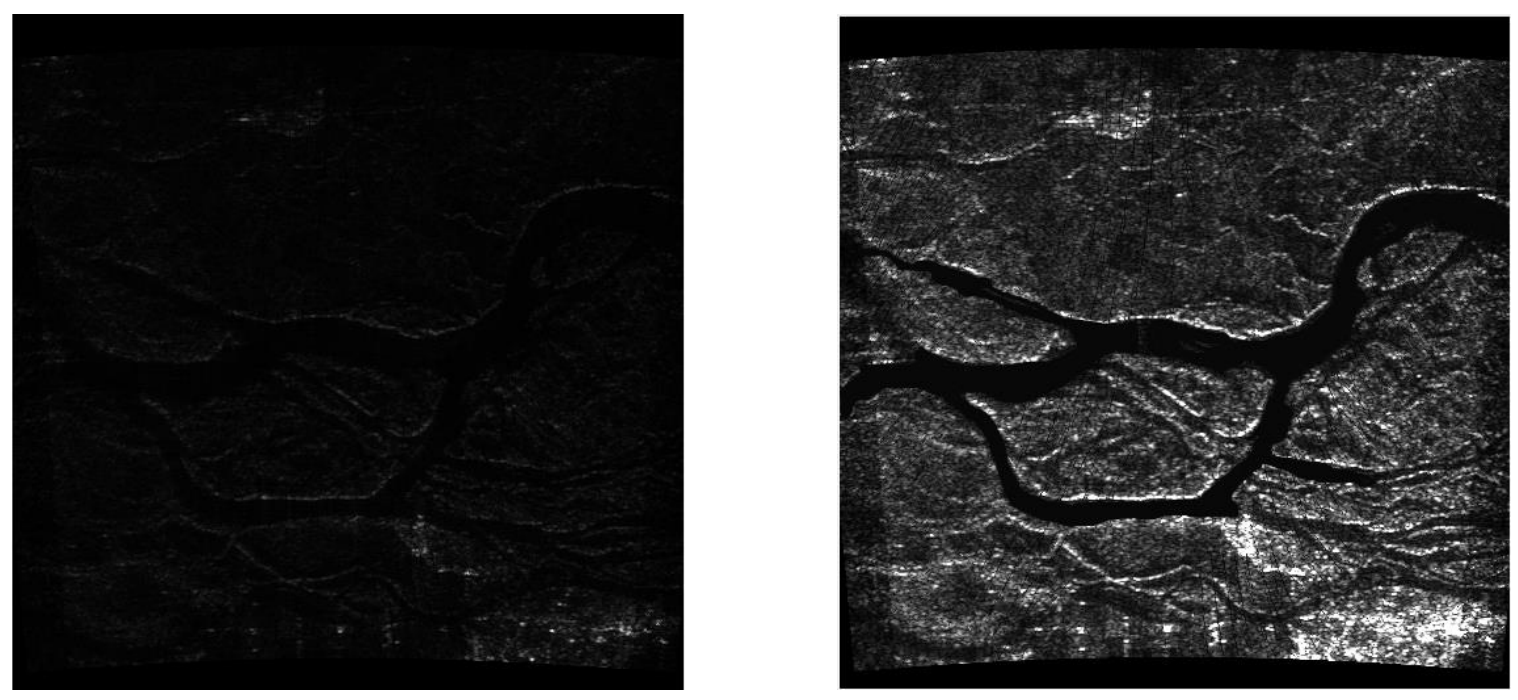

Figure 1. The result of linear (on the left) and statistical (on the right) matching of the dynamic ranges of received radar signal and output image

\section{Non-Linear Approach to the Matching Of Dynamic Ranges: The Logarithm and Gamma Correction, Retinex Algorithm}

Algorithms such as logarithm, gamma correction and Retinex are widely used to increase the visibility of details in poorly lit areas of digital images. All of them establish the relationship between the input range of the signal and the output range of the displayed image in a nonlinear manner. In logarithm correction, the dependence of the each pixel's brightness in the radar image on the power of the signal reflected from the corresponding area of the earth's surface is set by the expression:

$J(x, y)=\frac{\log \left(P_{r}(x, y)\right)}{\log \left(P_{r} \max \right)} \cdot J \max$

where $P_{r}$ max is maximum received signal power, Jmax - maximum brightness of the display device. The gamma correction algorithm (Johnson, 2007) is based on power transformation:

$\mathrm{J}(\mathrm{x}, \mathrm{y})=\mathrm{A} \cdot\left(\mathrm{P}_{\mathrm{r}}(\mathrm{x}, \mathrm{y}) / \mathrm{P}_{\mathrm{r}} \max \right)^{\gamma} \cdot \mathrm{Jmax}$

where $A$ is a factor, $\gamma$-gamma parameter. Typically, $A$ is assumed to be one, and the standard gamma value for RGB color spaces is 2.2 (Munson and Cox, 2013). Using both logarithm and gamma correction, the intensity of the dark pixels in the image increases more than the intensity of the light pixels. This improves the recognition of parts in poorly lit areas. An example of the use of both algorithms in the formation of radar images is shown in Figure 2.

As can be seen from the figure, logarithmic and power-law matching of dynamic ranges allows increasing the visibility of radio-contrast objects on the background of the water surface by increasing their local contrast. However, the level of local contrast in the brightest areas has significantly decreased. As a result, objects with large RCS merged into large illuminated "spots"; their visibility on the surrounding background was reduced. These undesirable effects are due to 
International Journal of Mathematical, Engineering and Management Sciences

Vol. 4, No. 6, 1448-1458, 2019

https://dx.doi.org/10.33889/IJMEMS.2019.4.6-114

the fact that the above algorithms have a single-point effect. They don't take into account the local neighborhood of pixels; therefore, in cases where the image contains both strongly dark and strongly light local areas, these algorithms can't provide high-quality matching of dynamic ranges. To overcome these problems, the Retinex algorithm is usually used.
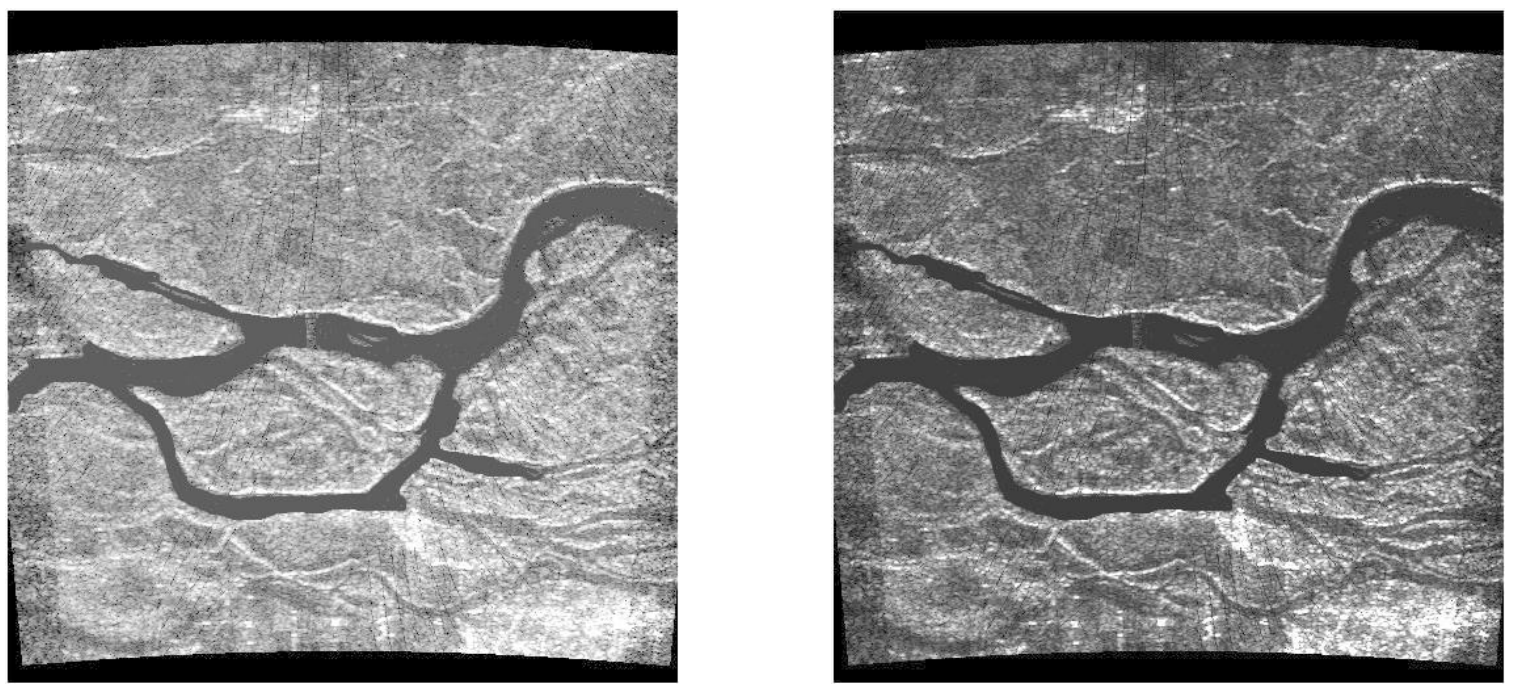

Figure 2. The result of logarithmic (on the left) and power-law (on the right) matching of the dynamic ranges of received radar signal and output image

Retinex algorithm, as a model of vision to compensate the variability of external lighting in images, was first proposed for the processing of optical images in (Land and McCann, 1971). Retinex is a vision model based on a simplified simulation of the biological structure of the visual tract of animals. The main advantage of the Retinex model is the mechanism of dynamic compression of image lighting, which allows to register bright and darkened areas of the image that are lost during the transition from the natural dynamic range to a fixed range of image representation in the computer. Below we briefly describe the algorithm. The image perceived by the sensors can be represented as:

$\mathrm{J}=\mathrm{L} \cdot \mathrm{R}$

where $\mathbf{J}$ is the measured intensity, $\mathbf{L}$ - lighting, $\mathbf{R}$ - reflectivity of the surface of the image element. For radar images, the presence of areas with significantly different RCS in the frame can be interpreted as lighting irregularity. In its simplest form, the Retinex algorithm is expressed as follows:

$\mathrm{J}=\mathrm{L} \cdot \mathrm{R} \rightarrow \mathrm{j}=\mathrm{l}+\mathrm{r}$

where $\boldsymbol{j}=\log (\mathrm{J}), \boldsymbol{l}=\log (\mathbf{L}), \boldsymbol{r}=\log (\mathbf{R})$. Estimation $\widetilde{R}$ is derived from the expression

$\tilde{\mathrm{r}}=\mathrm{j}-\tilde{\mathrm{l}}$ 
International Journal of Mathematical, Engineering and Management Sciences

Vol. 4, No. 6, 1448-1458, 2019

https://dx.doi.org/10.33889/IJMEMS.2019.4.6-114

where $\tilde{l}$ is approximation of $l, \tilde{r}$ - estimation of $r$, by formula:

$\widetilde{\mathrm{R}}=\exp (\widetilde{\mathrm{r}})$

The main problem is to find an approximation for the lighting of the image, that is, to determine the function $\tilde{l}(\mathrm{x}, \mathrm{y})$ that meets the parameters of the original image using its representation (7) in the best manner. In recent years, the models of Retinex with heuristic function of approximation of lighting, called Single Scale Retinex (SSR) and its extension - Multi Scale Retinex (MSR), are the mostly applied. The SSR algorithm evens out the illumination of the image, maintaining local contrast in low-and high-light areas. The correction takes place according to the formula:

$\mathrm{R}(\mathrm{x}, \mathrm{y})=\log (\mathrm{J}(\mathrm{x}, \mathrm{y}))-\log (\mathrm{G}(\mathrm{x}, \mathrm{y}) * \mathrm{~J}(\mathrm{x}, \mathrm{y}))$

where $*$ is the convolution operation, $\mathrm{G}(\mathrm{x}, \mathrm{y})$ - the smoothing Gaussian function with blur radius r:

$\mathrm{G}(\mathrm{x}, \mathrm{y})=\frac{1}{2 \cdot \pi \cdot \mathrm{r}^{2}} \cdot \exp \left(-\frac{\mathrm{x}^{2}+\mathrm{y}^{2}}{2 \cdot \mathrm{r}^{2}}\right)$

MSR algorithm is defined by expression:

$\mathrm{R}(\mathrm{x}, \mathrm{y})=\sum_{\mathrm{m}} \mathrm{W}_{\mathrm{m}} \cdot\left[\log (\mathrm{J}(\mathrm{x}, \mathrm{y}))-\log \left(\mathrm{G}\left(\mathrm{x}, \mathrm{y}, \mathrm{r}_{\mathrm{m}}\right) * \mathrm{~J}(\mathrm{x}, \mathrm{y})\right)\right]$

where $m$ is the scale sequence number, $W_{m}$ - weighting factor for scale $m$. It is experimentally established that it is optimal to use 3 different scales. Using a larger number of scales in (13) significantly increases the amount of computation without significantly improving the resulting image processing (Jobson et al., 1997). Figure 3 shows the result of applying Retinex algorithm to the radar image.

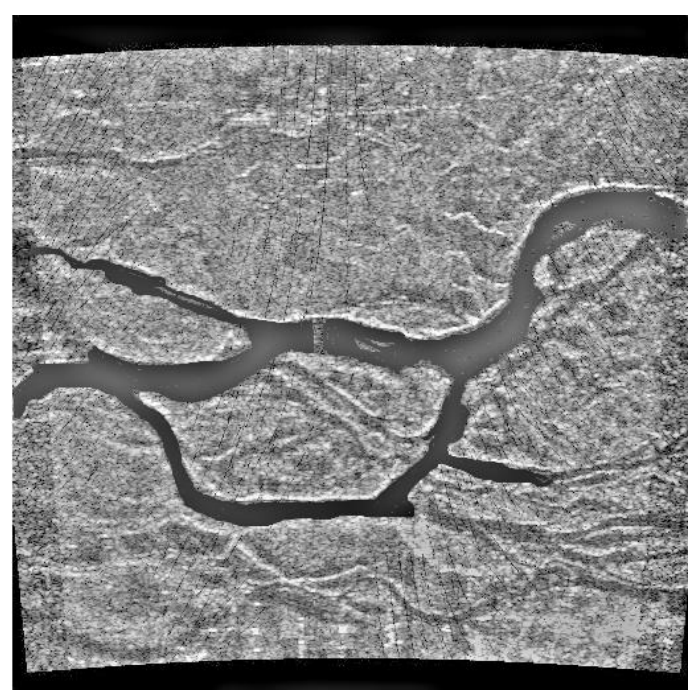

Figure 3. The result of Retinex algorithm application in the matching of the dynamic ranges of received radar signal and output image 
International Journal of Mathematical, Engineering and Management Sciences

Vol. 4, No. 6, 1448-1458, 2019

https://dx.doi.org/10.33889/IJMEMS.2019.4.6-114

As can be seen from Figure 3, Retinex shows better results in terms of increasing the visibility of radio-contrast objects on the water surface compared to procedures (5) and (6). This was achieved by taking into account the properties of the local neighborhood of each pixel. At the same time, local contrast in the brightest areas of the image is even worse. As a result of the global equalization of illumination, the observed objects are difficult to differentiate in terms of their reflectivity. This effect is greatly exacerbated by the amplification of speckle noise inherent in ground-reflected radar signal (Mather and Tso, 2009).

\section{Synthesis of the Algorithm Taking into Account the Peculiarities of Radar Images of the Earth's Surface}

As can be seen from Figures 1-3, none of the procedures discussed in section 4 provides an acceptable result in the radar image formation. However, this can be achieved by modifying and combining them as needed. Let's formulate the requirements for an algorithm that would provide a suitable result:

- the algorithm should increase the visibility of small radio-contrast objects on the background with low reflectivity;

- the algorithm should not significantly reduce the level of local contrast in the brightest areas of the image;

- objects with a low RCS should not look brighter than objects away with a large RCS;

- histogram of the generated image should correspond not normal, but the Rayleigh law of distribution: this factor is due to the theoretical basics of radiolocation (Pasmurov and Zinoviev, 2005; Kondratenkov and Frolov, 2005).

In order to ensure the most effective implementation of the first item from the above list, when forming a radar image, it is necessary to take into account the properties of the local neighborhoods of each element of the resolution. Thus, we choose the Retinex algorithm as the basic one. Write expression (11) in the form of the logarithm of the ratio:

$R(x, y)=\log \left(\frac{\mathrm{J}(\mathrm{x}, \mathrm{y})}{\mathrm{G}(\mathrm{x}, \mathrm{y}, r) * \mathrm{~J}(\mathrm{x}, \mathrm{y})}\right)$

The following two requirements from the list can be met by modifying (14) as follows:

$R(x, y)=\frac{\mathrm{J}(\mathrm{x}, \mathrm{y})}{\mathrm{G}(\mathrm{x}, \mathrm{y}, r) * \mathrm{~J}(\mathrm{x}, \mathrm{y})} \log (\mathrm{J}(\mathrm{x}, \mathrm{y}))$

Here, the logarithm of the ratio of initial intensity to lighting was eliminated to reduce the effect of local contrast degradation in bright areas of the image. The logarithm multiplier of the initial intensity counteracts the decrease of brightness of objects with large RCS. This allows them not to merge into large white "spots" and increases their visibility on the surrounding background.

To describe the amplitude fluctuations of the radio signal in radar, the Rayleigh distribution is used (Barton, 2013), which has a density

$f\left(i ; \sigma_{r}\right)=\frac{i}{\sigma_{r}^{2}} \exp \left(-\frac{i^{2}}{2 \cdot \sigma_{r}^{2}}\right)$ 
International Journal of Mathematical, Engineering and Management Sciences

Vol. 4, No. 6, 1448-1458, 2019

https://dx.doi.org/10.33889/IJMEMS.2019.4.6-114

with a single scale parameter $\sigma_{r}$. The mathematical expectation for this distribution is determined by the expression

$\mu_{r}=\sqrt{\frac{\pi}{2} \sigma_{r}}$

To provide the histogram of the formed radar image with a distribution as close as possible to the Rayleigh one, we present the result of $R(x, y)$, obtained at step (15), to the range $\left(0 \ldots 3 \sigma_{r}\right)$. The scale parameter is defined from (17):

$\sigma_{r}=2 \frac{{\widetilde{\mu_{r}}}^{2}}{\pi}$

where $\widetilde{\mu_{r}}$ is the estimation of mathematical expectation of $R(x, y)$ :

$\widetilde{\mu_{r}}=\frac{1}{\mathrm{~N}} \cdot \sum_{x, y} R(x, y)$

\section{Results \& Discussion}

The radar image formed according to the formulas (15-19) and its histogram are shown in Figure 4. The result meets all the requirements from the list given in the previous section. Objects on the background of the water surface are visually distinguishable; the level of local contrast within the entire image is high enough; the effect of reducing the brightness of objects with large RCS is minimized.
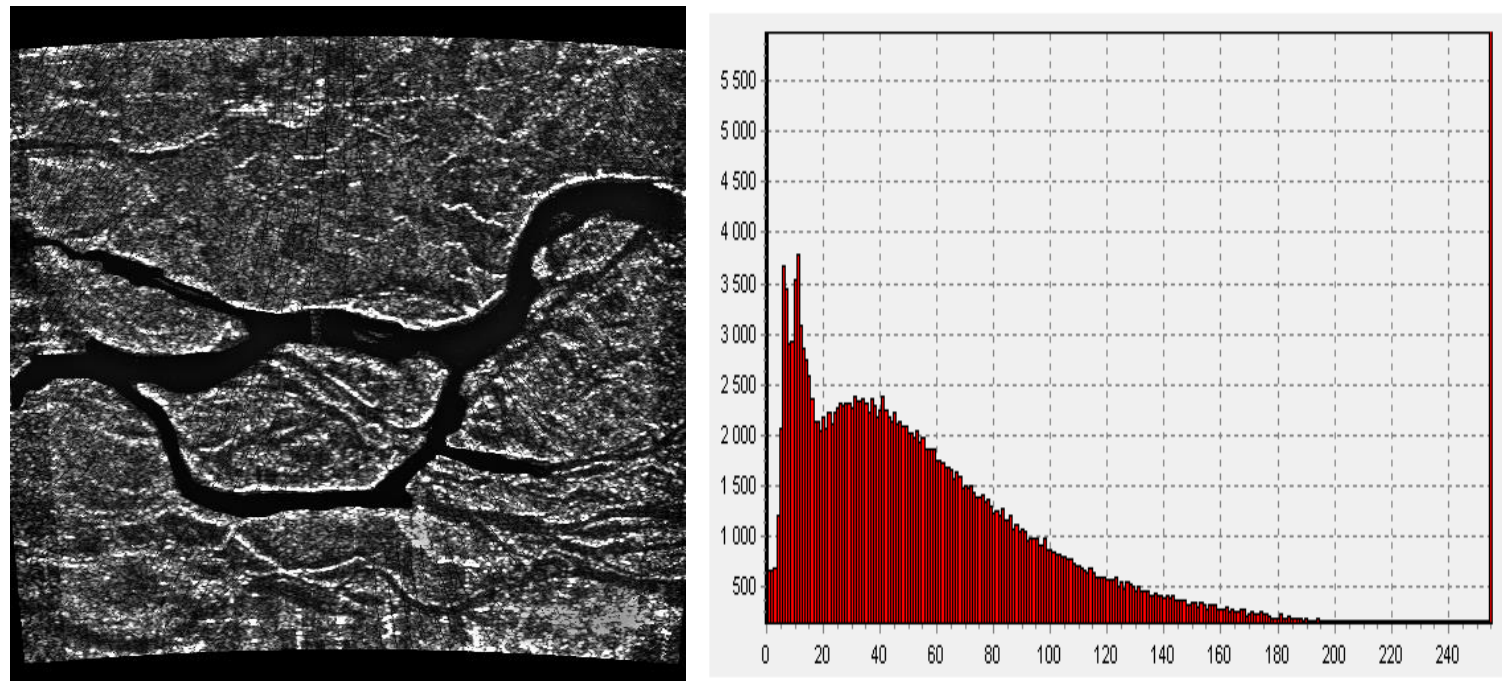

Figure 4. Result of application of the proposed algorithm in the formation of radar image (on the left) and the histogram of the image (on the right)

Signal processing in modern airborne radars is digital. The signal received by antenna is subject to analog-to-digital conversion. The further processing and visualization in the form of two- 
International Journal of Mathematical, Engineering and Management Sciences

Vol. 4, No. 6, 1448-1458, 2019

https://dx.doi.org/10.33889/IJMEMS.2019.4.6-114

dimensional image is performed using the embedded computer system. Airborne computing machines are characterized by limited performance. But at the same time they should provide signal processing and image formation in real time. Therefore, reducing the time spent on matching the dynamic ranges of received signal and visualization device is an urgent problem. Two aspects of optimization can be distinguished for expression (15): the computation of the logarithm by the tabular method and the implementation of fast Gaussian blur by the infinite impulse response (IIR) filter (van Vliet et al., 1998). Thus, when using piecewise linear approximation of the logarithm in combination with a third-order IIR-filter, only 19 floating-point multiplication operations are required for each element of the resolution. This is quite feasible for embedded airborne computer system.

\section{Conclusion}

This article proposes a method of matching the dynamic ranges of radar signal reflected by the earth's surface, and visualization devices. The method is based on adaptive nonlinear signal processing. Adaptability is achieved by taking into account the local neighborhood properties of each resolution element. The proposed method provides better local contrast of radar images and improves discrimination of individual objects, the form of which is familiar to the operator. The computational complexity of the proposed algorithm is optimized and allows its real-time implementation. The proposed method is recommended for use in embedded computer system as part of airborne radars.

\section{Conflict of Interest}

The authors confirm that there is no conflict of interest to declare for this publication.

\section{Acknowledgement}

The work was carried out in accordance with the decree of the Government of the Russian Federation from 09.04.2010 № 218 (PROJECT 218) in the framework of R\&D, executing with the financial support of the Ministry of education and science of the Russian Federation (agreement № 074-11-2018-025 from 13.07.2018). Work is performed in lead R \& D performer organization: Federal State Budgetary Educational Institution of Higher Professional Education BSTU "VOENMEH" named after D.F. Ustinov.

The research carried out with the financial support of the grant from the Program Competitiveness Enhancement of Peter the Great St. Petersburg Polytechnic University.

\section{References}

Barton, D.K. (2013). Radar equations for modern radar. 1st Edition. Artech House Radar.

Bataev, A.V. (2019) Efficiency estimation model of 3D technology in the construction industry. In 2019 IEEE Conference of Russian Young Researchers in Electrical and Electronic Engineering, ElConRus 2019, art. no. 8657181, pp. 1376-1381. doi: 10.1109/EIConRus.2019.8657181.

Davydov, V.V., Dudkin, V.I., \& Myazin, N.S. (2016). Nutation line shape for the nonstationary regime of magnetic resonance flowmeter-relaxometer. Journal of Communications Technology and Electronics, 61(10), 1159-1165. doi:10.1134/S1064226916100077. 
International Journal of Mathematical, Engineering and Management Sciences

Vol. 4, No. 6, 1448-1458, 2019

https://dx.doi.org/10.33889/IJMEMS.2019.4.6-114

EUROCAE ED-179 (2011). Minimum aviation system performance standard (MASPS) for enhanced vision systems, synthetic vision systems, combine vision systems and enhanced flight vision systems. The European Organisation for Civil Aviation Equipment.

Galeeva, M.A., Baranov, M.A., Pavlov, V.A., Velichko, E.N., Zavjalov, S.V., Govorov, I.E., Pervunina, T.M., \&. Komlichenko, E.V. (2019) On specific features of the endoscopic image processing. Journal of Physics: Conference Series, 1236 (1), art. no. 012036, doi: 10.1088/1742-6596/1236/1/012036.

Ivanov, S.I., Kyrnyshev, A.M., \& Lavrov, A.P. (2015). Measuring radar cross-section of complex-shaped objects using the doppler shift. Paper presented at the 2015 International Siberian Conference on Control and Communications, SIBCON 2015 - Proceedings (pp. 1-4). IEEE. Omsk, Russia. doi:10.1109/SIBCON.2015.7147075.

Ivanov, S.I., Lavrov, A.P., Saenko, I.I., Bessoltsev, S.A., Dostovalov, A.V., \& Wolf, A.A. (2018). Microwave photonic beamforming system with broadband chirped fiber bragg grating. Paper presented at the Proceedings of SPIE - the International Society for Optical Engineering, 10774. doi:10.1117/12.2318086.

Jobson, D.J., Rahman Z., \& Woodell, G.A. (1997). A multiscale retinex for bridging the gap between color images and the human observation of scenes. IEEE Transactions Image Process 6(7), 965-976.

Johnson, C. (2007). The practical zone system: for film and digital photography. 4th Edition. Boston, Focal Press.

Kaasik, V.P. \& Rogov, S.A. (2016). Comparison of the operation of an acousto-optic spectrum analyzer and an acousto-optic pseudo-wigner processor by analyzing the time-frequency distributions of frequencymodulated signals. Journal of Optical Technology (A Translation of Opticheskii Zhurnal), 83(5), 290294. doi:10.1364/JOT.83.000290.

Klochkov, Y., Gazizulina, A., Golovin, N., Glushkova, A., \& Zh, S. (2018). Information model-based forecasting of technological process state. Paper presented at the 2017 International Conference on Infocom Technologies and Unmanned Systems: Trends and Future Directions, (ICTUS 2017) (pp. 709712).IEEE. ADET, Amity University Dubai, UAE. doi:10.1109/ICTUS.2017.8286099.

Kondratenkov, G.S., \& Frolov, A.Y. (2005). Radio broadcasting. Radar systems for remote sensing of the Earth. Textbook for high schools. Moscow, Radiotekhnika.

Korobeynikov, A.G., Grishentsev, A.Yu., Velichko, E.N., Korikov, C.C., Aleksanin, S.A., Fedosovskii, M.E., \& Bondarenko, I.B. (2016). Calculation of regularization parameter in the problem of blur removal in digital image. Optical Memory and Neural Networks (Information Optics), 25(3), 184-191. doi:10.3103/S1060992X16030036.

Land, E.H., \& McCann, J.J. (1971). Lightness and retinex theory. Journal of the Optical Society of America. 61(1), 1-11.

Logunov, S.E., Davydov, V.V., Vysoczky, M.G., \& Titova, O.A. (2018). Peculiarities of registration of magnetic field variations by a quantum sensor based on a ferrofluid cell. Journal of Physics: Conference Series, 1135(1), art. no. 012069. doi: 10.1088/1742-6596/1135/1/012069.

Mather, P., \& Tso, B. (2009). Classification methods for remotely sensed data. second edition. CRC Press, Taylor and Francis Group, Boca Raton, USA.

Matveev, S.A., Bizov, A.N., Bistrov, S.Yu., Garmash, V.N., Isenko, S.I., Korobochkin, D.M., Petrov, Yu.V., Rudika, S.A., Strahov, S.Yu., \& Sircev, A.N. (2018). Helicopter system that provide information support for safety of flights and conduct search and rescue operations. Bulletin of the Kyrgyz-Russian Slavic University, 18(9), 60-64. 
International Journal of Mathematical, Engineering and Management Sciences

Vol. 4, No. 6, 1448-1458, 2019

https://dx.doi.org/10.33889/IJMEMS.2019.4.6-114

Meena, D., \& Prakasam, L.G.M. (2008). FPGA based real time solution for sensitivity time control. In 4th IEEE International Symposium on Electronic Design, Test and Applications (delta 2008) (pp. 244-248). IEEE. Hong Kong, China.

Munson, J., \& Cox, F. (2013). Video circuit collection. In Analog Circuit Design. Vol. 2. Immersion in the Black Art of Analog Design Chapter 34 (pp. 769-800). Editors: Dobkin, B. \& Williams, J. Elsevier.

Pasmurov, A.Y., \& Zinoviev, J.S. (2005). Radar imaging and holography. IET radar, sonar and navigation series 19. London, United Kingdom.

Privalov, V.E., \& Shemanin, V.G. (2017). Estimation of the error of lidar measurements of atmospheric radionuclide concentrations. Measurement Techniques, 60(9), 962-967. doi:10.1007/s11018-017-13015.

Privalov, V.E., \& Shemanin, V.G. (2018). Monitoring hydrogen sulfide molecules in the atmospheric boundary layer by differential absorption and scattering lidar from space. Journal of Optical Technology (A Translation of Opticheskii Zhurnal), 85(4), 229-232. doi:10.1364/JOT.85.000229.

Shang, W., Xu, Y., Qi, J., Xue, W., \& Makarov, S.B. (2017). Optimal waveform of the partial-respond signal based on minimum out-of-band radiation criterion. Applied Sciences (Switzerland), 7(10), 1-12 doi:10.3390/app7101086.

Shelton, K.J., Kramer, L.J., Ellis, K., \& Rehfeld, S.A. (2012). Synthetic and enhanced vision systems (sevs) for nextgen simulation and flight test performance evaluation. In 2012 IEEE/AIAA 31 st Digital Avionics Systems Conference (DASC) (pp. 2D5-1). IEEE. Williamsburg, VA, USA.

Skolnic, M.I. (1990). Radar handbook. 2nd Edition. McGraw-Hill Professional.

Tarasenko, M.Yu., Davydov, V.V., Lenets, V.A., Akulich, N.V., \& Yalunina, T.R. (2017) Features of use direct and external modulation in fiber optical simulators of a false target for testing radar station. Lecture Notes in Computer Science (including subseries Lecture Notes in Artificial Intelligence and Lecture Notes in Bioinformatics), 10531 LNCS, pp. 227-232 DOI: 10.1007/978-3-319-67380-6_21.

van Vliet, L.J., Young, I.T., \& Verbeek, P.W. (1998). Recursive Gaussian derivative filters. In proceedings of Fourteenth International Conference on Pattern Recognition (Cat. No.98EX170). IEEE. Brisbane, Queensland, Australia.

Vygolov, O.V. (2013). Enhanced and synthetic vision systems development based on integrated modular avionics for civil aviation. IEEE/AIAA 32st Digital Avionics Systems Conference (DASC'2013), 2B5.12B5.13. IEEE. East Syracuse, NY, USA.

Zheltov, S., \& Vygolov, O.V. (2015). Enhanced, synthetic and combined vision technologies for civil aviation. In Computer Vision in Control Systems-2 (pp. 201-230). Springer, Cham.

Ziniakov, V.Y., Gorodetskiy, A.E., \& Tarasova, I.L. (2016). Control of vitality and reliability analysis. In Smart Electromechanical Systems (pp. 193-204). Springer, Cham. doi:10.1007/978-3-319-27547-5_18. 